\title{
Studying the Effect of Building Block Shape on Sierpinski Tetrahedron Fractal Antenna Behavior Using FDTD-Equivalent Electric Circuits
}

\author{
A. N. Jabbar, \\ University of Babylon, Hilla, Babylon, Iraq,e-mail: Ahmed.Najah72@Yahoo.com
}

\begin{abstract}
This research aims to study the effect of the modification in the Building Block Shape (BBS) on the frequency response of Sierpinski three dimensional pre-fractal antenna using conventional circuit analysis. Three different shapes of BBS's are used to build the antenna. The surface current is analyzed and compared for every BBS. A simple model was devised using the nodal analysis to provide an iterative method for calculating the distribution of currents and voltages for the antenna at every node. The antennas parameters such as Y-parameter, Input Return Loss (IRL), delivered power and surface current are investigated at the first and third tuning frequencies to enhance the perspective of the BBS effect.
\end{abstract}

Index Terms - Fractal antenna, antenna design, modeling and measurements, numerical techniques, electric circuits, FDTD.

\section{INTRODUCTION}

The fractal is a fascinating entity that is spread widely in nature. Despite of the fractal complicated shape, it starts with a very simple shape like a line or square and then it is iterated, theoretically, to infinity to create a fractal $[1,2]$. The infinite iterations are neither practical nor realizable thus the number of iterations should be limited to a reasonable value. The generated shape is called a prefractal to differentiate it from the fractal which is created using infinite level of iterations.

The pre-fractal is heavily invested in antenna design and microwave devices because it is space compact, multi-band and it has many other attractive features that make it an eligible choice for various microwave applications [3-10]. Therefore, we need to study the electromagnetic properties of the fractal. Hence, we need a simple iterative model to explore their behavior. A remarkable approach is the nodal analysis which is used to analyze ordinary electric circuits [11, 12, and 13]. This approach is adopted by many researchers to anticipate the frequency response of a certain shape.

One of the earliest attempts to build a recursive model was presented by [14]. In this paper each element was replaced by its S-parameter block to construct an iterative model. This model was implemented by [15] to study the effect of changing the flare angle on the antenna behavior.

The first electrical model was proposed by [16]. The author suggested that each area can be replaced by its equivalent RLC circuit to study the Sierpinsky fractal antenna properties. The author in [17] modified the electric model to construct a new fast accurate model. In [18], the author substituted the conventional Sierpinsky fractal antenna by wire elements depending on the electrical circuit to 
prove the model proposed by [16]. The nodal analysis was implemented by [19] to create a matrix that can be used to represent the antenna under study. This approached was used by [20] to replace the Sierpinski carpet by its equivalent gasket fractal antenna. This model was generalized by [21] to study the Sierpinsky tetrahedron antenna. In [22], the author compared between the study given by [21] and the practical results. The results proved the accuracy of the electrical model. Hence, it was the the topic for modeling and development for other researchers [23, 24].

In all the above studies the effect of the BBS was not studied. The contribution of this research is to study and analyze the effect of the BBS modification on the antenna performance. This aspect is important because it gives us the necessary clues on how to tailor a certain BBS to re-route the surface currents in order to achieve the desired antenna response. This paper suggests two modified block shapes. The first is created by altering the original block geometry while the second is created by adding parasitic elements. This process will result in a new surface current distribution leading to a new behavior. The nodal analysis is used as a tool for the analysis of this study.

\section{From EleCtromagnetics TO CiRCUIT THEORY}

The FDTD is an important numerical approach used to solve the Maxwell field equations. It works as shown in Fig.1 [25]

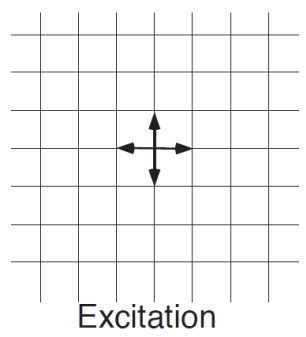

Excitation

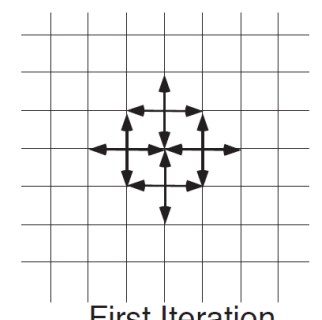

First Iteration

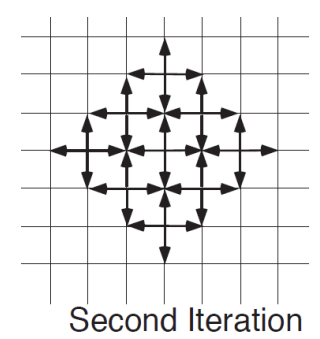

Second Iteration

Fig.1 FDTD grid progress

In the beginning, the algorithm starts by dividing the whole structure into a grid of cells. Then the system is excited using a known source. Maxwell equation, given in Eq.1, is then solved at the boundaries of the first cell. The results are then used as driving potentials for the next cells and so on. After completing the solution for all cells the algorithm finishes its calculations [26, 27].

$$
\left[\begin{array}{c}
\nabla \times \vec{H} \\
-\nabla \times \vec{E}
\end{array}\right]=\left[\begin{array}{l}
\overrightarrow{J_{e}} \\
\overrightarrow{J_{m}}
\end{array}\right]+\frac{\partial}{\partial t}\left[\begin{array}{l}
\vec{D} \\
\vec{B}
\end{array}\right]
$$

where $H$, and $E$ are the magnetic and electric fields, $J_{e}$ and $J_{m}$ are the voltage and current densities and $D$ and $B$ are the electric field and magnetic flux densities.

Assume we have a cell in which the propagation occurs along one dimension (along the $\mathrm{x}$-axis) as shown in Fig. 2 


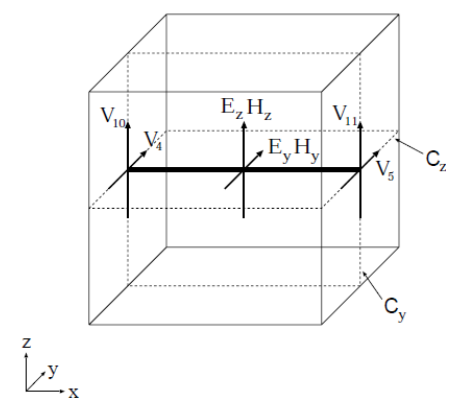

Fig.2 One dimensional propagation within a cell

The electric field propagation is given in Eq.2 [25]

$$
\frac{\partial^{2} E}{\partial^{2} x}-\left(\frac{1}{v}\right)\left(\frac{\partial^{2} E}{\partial^{2} t}\right)-\sigma \mu \frac{\partial E}{\partial t}=0
$$

where $v, \sigma$ and $\mu$ are the propagation velocity, conductivity and permeability respectively. By comparing Eqn. 2 with the equation that describes the voltage propagation along a one dimensional transmission line, given by Eq.3, we can notice the similarities between them [28, 29]

$$
\frac{\partial^{2} V}{\partial^{2} x}-(L C)\left(\frac{\partial^{2} V}{\partial^{2} t}\right)-L G \frac{\partial V}{\partial t}=0
$$

where $V, L, C$ and $G$ are the voltage, inductance, capacitance and admittance of a lossy transmission line respectively.

Hence we can convert the one-dimensional cell shown in Fig.2 to its equivalent electric circuit shown in Fig.3. The distributed element can be substituted by equivalent impedance as shown in Fig.2b.

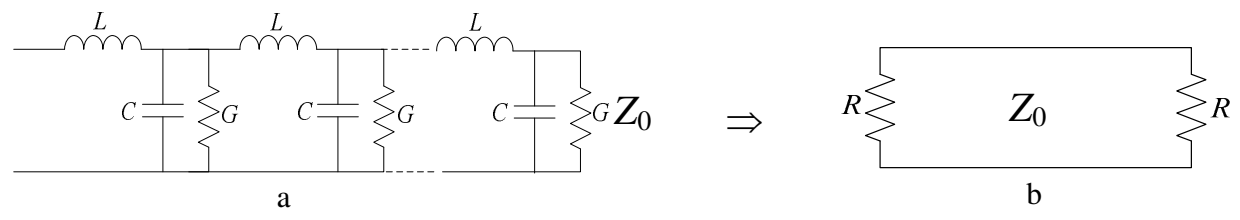

Fig.3 one-dimensional cell equivalent electric circuit, a) cell, b) reduced electric circuit

where $Z_{0}=(\mu / \varepsilon)^{\frac{1}{2}}=(L / C)^{\frac{1}{2}}$. This method can be extended for two dimensional cells to describe the electric and magnetic fields over a surface as shown in Fig.4.

Here, $V_{i}$ represents the voltage of the $i$-th cell and $R$ and $Z_{0}$ are total impedances seen at the edges. We conclude that the equivalent impedance is independent of the surface topology and is related only to the area itself and the type of material.

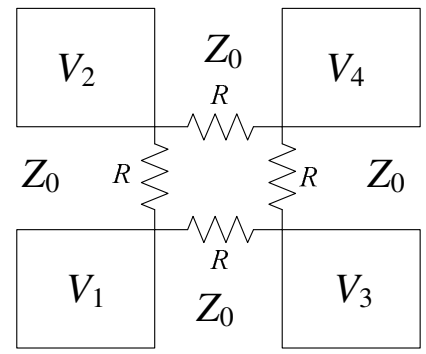

Fig.4 equivalent electric circuit of the two dimensional cell 
After converting the shape into a simple electric circuit, we need to fully analyze the structure. Any electric circuit can be identified completely by its voltages and currents. Hence, we can use the nodal analysis to represent KCL and KVL as a matrix using circuit graph theory. This method transforms the electric circuit into a matrix to calculate the required voltages (electric field) and the currents (magnetic field) passing through the structure under analysis [8].

Three different BBS are proposed for purpose of study as shown in Fig. 5.The analysis given previously will be used to represent each BBS by an equivalent electric circuit.

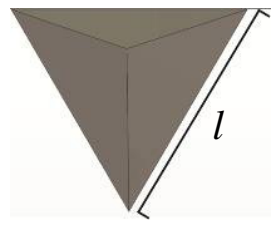

(a)

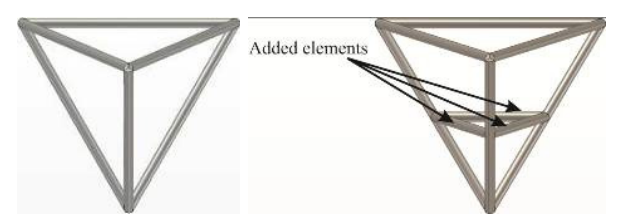

(b)

(c)

Fig.5 The BBS shapes, a. Solid BBS, b. Cylindrical BBS, c. Modified Cylindrical BBS

For the three BBSs, the length of their edges is $l=12.25 \mathrm{~mm}$. The solid BBS has four surfaces with an area equals to $A$. It can be divided into three equal parts. Each part can be replaced by an equivalent $Y$ as shown in Fig. 6.a. The edges of the four surfaces are connected in parallel yielding an equivalent admittance $2 Y$. By applying the same approach in $[24,30]$ we have the equivalent electric circuit shown in Fig. 6 b.

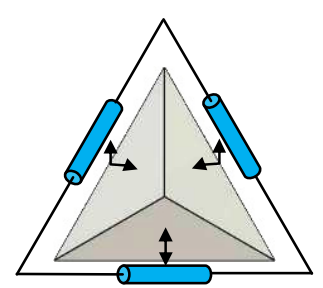

(a)

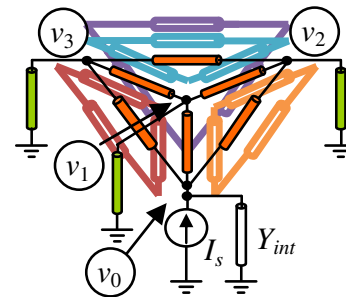

(b)

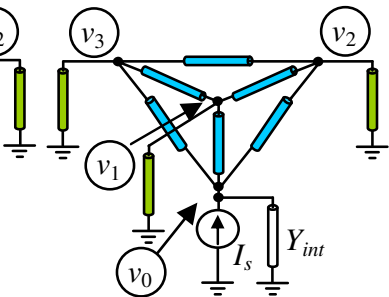

(c)

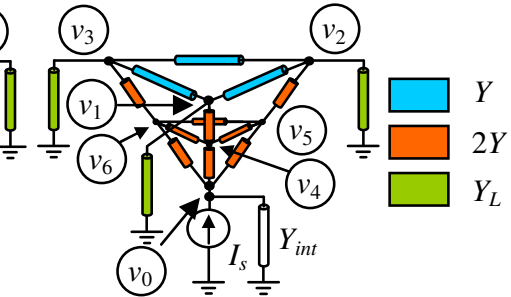

(d)

Fig. 6 The equivalent electric circuit for, a. Area equivalent impedance, b. Solid BBS, c. Cylindrical BBS, d. Modified Cylindrical BBS

For the cylindrical BBS, the diameter $d$ is set to $0.56 \mathrm{~mm}$ to ensure that its surface area equals to $A / 3$ in order to be replaced by an admittance $Y$. The equivalent electric circuit is shown in Fig. 6c. For the modified cylindrical BBS, the added elements are passing through the middle of each two adjacent edges; hence, their length equals to $l / 2$. Therefore, the admittance of these elements is $2 Y$. The equivalent electric circuit is shown in Fig. 6d.

Let us assume that the system is working at maximum power transfer condition then $Y_{L}=Y_{\text {int }}$, where $Y_{\text {int }}$ is the source internal admittance and $Y_{L}$ is the load admittance $\left(Z_{L}=50 \Omega\right)$. By using the nodal analysis, the $Y$ matrix for each BBS is given by Eq.4, Eq.5 and Eq.6.

For the solid BBS

$$
\mathbf{Y}=Y\left[\begin{array}{cccc}
6 & -2 & -2 & -2 \\
-2 & 6 & -2 & -2 \\
-2 & -2 & 6 & -2 \\
-2 & -2 & -2 & 6
\end{array}\right]+Y_{L} \mathbf{I}
$$


where $\mathbf{I}$ is a square identity matrix and $Y_{L} \mathbf{I}$ represents the grounded admittances part.

For the cylindrical BBS, we have

$$
\mathbf{Y}=Y\left[\begin{array}{cccc}
3 & -1 & -1 & -1 \\
-1 & 3 & -1 & -1 \\
-1 & -1 & 3 & -1 \\
-1 & -1 & -1 & 3
\end{array}\right]+Y_{L} \mathbf{I}
$$

And for the modified cylindrical BBS, we have

$$
\mathbf{Y}=Y\left[\begin{array}{ccccccc}
6 & 0 & 0 & 0 & -2 & -2 & -2 \\
0 & 4 & -1 & -1 & -2 & 0 & 0 \\
0 & -1 & 4 & -1 & 0 & -2 & 0 \\
0 & -1 & -1 & 4 & 0 & 0 & -2 \\
-2 & -2 & 0 & 0 & 8 & -2 & -2 \\
-2 & 0 & -2 & 0 & -2 & 8 & -2 \\
-2 & 0 & 0 & -2 & -2 & -2 & 8
\end{array}\right]+Y_{L} \mathbf{I}
$$

The source current $\mathbf{I}_{s}$ vector for the solid and cylindrical BBS (Fig.6b, c) is given in Eq.7

$$
\mathbf{I}_{s}=\left[\begin{array}{c}
I_{s} \\
0 \\
0 \\
0
\end{array}\right]
$$

While for the modified cylindrical BBS (Fig.6.d) is

$$
\mathbf{I}_{s}=\left[\begin{array}{c}
I_{s} \\
0 \\
0 \\
0 \\
0 \\
0 \\
0
\end{array}\right]
$$

Using Ohm's law, we can find the voltages at each node as follows:

$i$. For the solid BBS

$$
\mathbf{V}=\mathbf{Y}^{-1} \mathbf{I}_{s} \Rightarrow\left[\begin{array}{c}
v_{0} \\
v_{1} \\
v_{2} \\
v_{3}
\end{array}\right]=I_{s}\left[\begin{array}{c}
\frac{2 Y+Y_{L}}{Y_{L}^{2}+8 Y Y_{L}} \\
\frac{2 Y}{Y_{L}^{2}+8 Y Y_{L}} \\
\frac{2 Y}{Y_{L}^{2}+8 Y Y_{L}} \\
\frac{2 Y}{Y_{L}^{2}+8 Y Y_{L}}
\end{array}\right]
$$

ii. For the cylindrical BBS 
iii. For the modified cylindrical BBS

$$
\mathbf{V}=\mathbf{Y}^{-1} \mathbf{I}_{s} \Rightarrow\left[\begin{array}{c}
v_{0} \\
v_{1} \\
v_{2} \\
v_{3}
\end{array}\right]=I_{s}\left[\begin{array}{c}
\frac{Y+Y_{L}}{Y_{L}^{2}+4 Y Y_{L}} \\
\frac{Y}{Y_{L}^{2}+4 Y Y_{L}} \\
\frac{Y}{Y_{L}^{2}+4 Y Y_{L}} \\
\frac{Y}{Y_{L}^{2}+4 Y Y_{L}}
\end{array}\right]
$$

$$
\mathbf{V}=\mathbf{Y}^{-1} \mathbf{I}_{s} \Rightarrow\left[\begin{array}{c}
v_{0} \\
v_{1} \\
v_{2} \\
v_{3} \\
v_{4} \\
v_{5} \\
v_{6}
\end{array}\right]=I_{s}\left[\begin{array}{l}
\frac{21 Y Y_{L}-14 Y^{2}+5 Y_{L}^{2}}{11 Y Y_{L}^{2}-156 Y^{2} Y_{L}-460 Y^{3}+5 Y_{L}^{3}} \\
\frac{56 Y^{2}+10 Y Y_{L}}{11 Y Y_{L}^{2}-156 Y^{2} Y_{L}-460 Y^{3}+5 Y_{L}^{3}} \\
\frac{76 Y^{2}+20 Y Y_{L}}{11 Y Y_{L}^{2}-156 Y^{2} Y_{L}-460 Y^{3}+5 Y_{L}^{3}} \\
\frac{56 Y^{2}+10 Y Y_{L}}{11 Y Y_{L}^{2}-156 Y^{2} Y_{L}-460 Y^{3}+5 Y_{L}^{3}} \\
\frac{46 Y^{2}+33 Y Y_{L}+5 Y_{L}^{2}}{11 Y Y_{L}^{2}-156 Y^{2} Y_{L}-460 Y^{3}+5 Y_{L}^{3}} \\
\frac{96 Y^{2}+68 Y Y_{L}+10 Y_{L}^{2}}{11 Y Y_{L}^{2}-156 Y^{2} Y_{L}-460 Y^{3}+5 Y_{L}^{3}} \\
\frac{46 Y^{2}+33 Y Y_{L}+5 Y_{L}^{2}}{11 Y Y_{L}^{2}-156 Y^{2} Y_{L}-460 Y^{3}+5 Y_{L}^{3}}
\end{array}\right]
$$

The important values in Eq.11 are $v_{0}, v_{1}, v_{2}$ and $v_{3}$ which are given in Eq.12. These nodes are important because they represent the feeding point for the next stage.

The other points $\left(v_{4}, v_{5}\right.$, and $\left.v_{6}\right)$ are only intermediate points located between the source and the connection points.

$$
\left[\begin{array}{c}
v_{0} \\
v_{1} \\
v_{2} \\
v_{3}
\end{array}\right]=i_{s}\left[\begin{array}{l}
\frac{21 Y Y_{L}-14 Y^{2}+5 Y_{L}^{2}}{11 Y Y_{L}^{2}-156 Y^{2} Y_{L}-460 Y^{3}+5 Y_{L}^{3}} \\
\frac{56 Y^{2}+10 Y Y_{L}}{11 Y Y_{L}^{2}-156 Y^{2} Y_{L}-460 Y^{3}+5 Y_{L}^{3}} \\
\frac{76 Y^{2}+20 Y Y_{L}}{11 Y Y_{L}^{2}-156 Y^{2} Y_{L}-460 Y^{3}+5 Y_{L}^{3}} \\
\frac{56 Y^{2}+10 Y Y_{L}}{11 Y Y_{L}^{2}-156 Y^{2} Y_{L}-460 Y^{3}+5 Y_{L}^{3}}
\end{array}\right]
$$

After finding the voltages and currents we can advance to the next stage. Each point $\left(v_{0}, v_{1}, v_{2}\right.$ and $v_{3}$ ) can be considered as new feeding sources for the next stage. Following the same steps $i, i i$, and iii above and applying Eq. 7 to 9 we can find the voltages and currents passing through every branch in that stage and so on. The iterated model can be visualized as in Fig.7. 


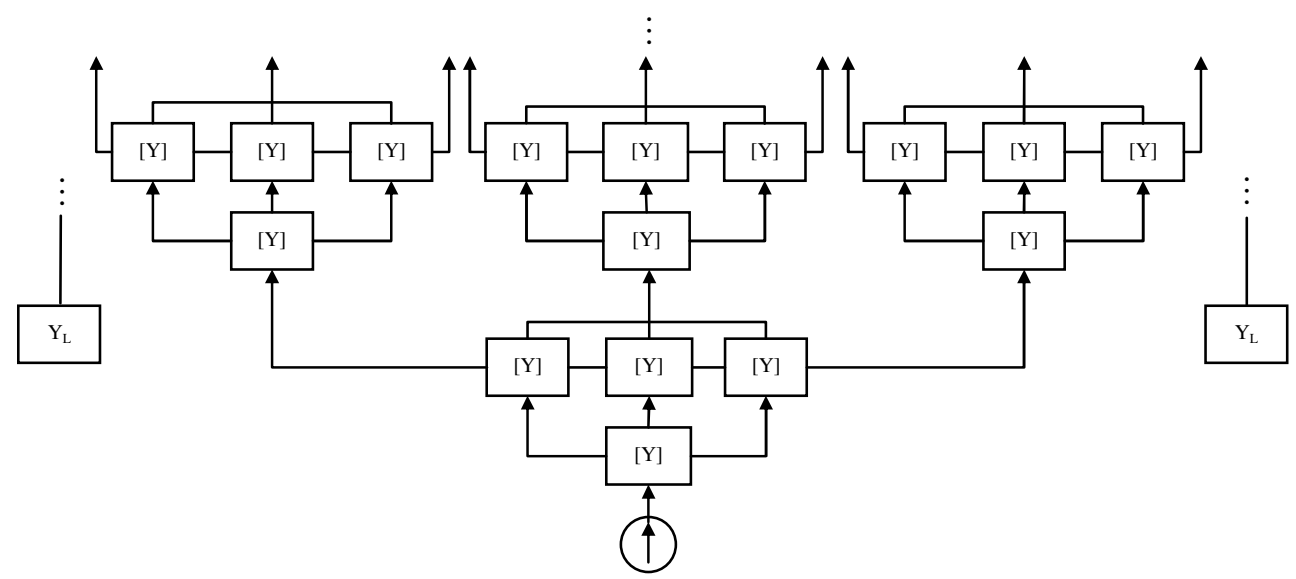

Fig. 7 The iterative model for the Sierpinski tetrahedron antenna

When all the required currents and voltages at every node are found, we can calculate the final $\mathbf{Y}$ parameters for the antenna using the fixed point asymptotic behavior depending on the iteration level $[31,32]$.

The Y-parameters can be used to calculate the other types of parameters (scattering, hybrid, transmission line...). Equation 13 can be used to calculate $\mathrm{S}$ parameters [33]

$$
\mathbf{S}=(\mathbf{Z}+\mathbf{I})^{-1}(\mathbf{Z}-\mathbf{I})
$$

where $\mathbf{Z}$

$$
\mathbf{Z}=\mathbf{Y}^{-1}
$$

Using the suitable set of parameters, we can calculate all the antenna parameters such as, the reflection coefficient, the Voltage Standing Wave Ratio VSWR as in Eq. 15 [34], and the delivered power as in Eq. 16 [35]

$$
\begin{gathered}
\operatorname{VSWR}=\frac{1+S_{11}}{1-S_{11}} \\
P_{D}=\left(1-S_{11}^{2}\right) \text { Watt }
\end{gathered}
$$

From the calculated voltages and currents at each node we can find the tuning frequencies, the electric field distribution, the surface current density, the electric energy, and the power flow.

\section{The Model SettingS AND Simulation Results}

The Sierpinski tetrahedron antennas are constructed using three iterations. For every BBS's the resulting antenna is shown in Fig. 8.

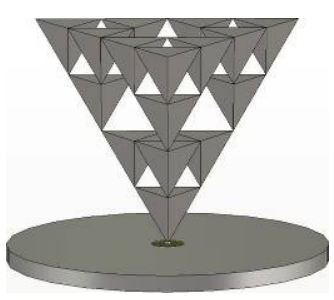

(a)

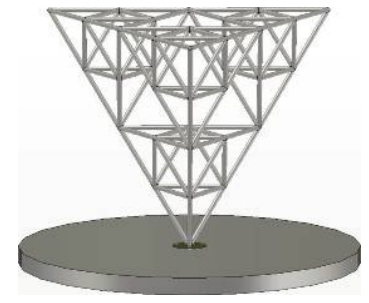

(b)

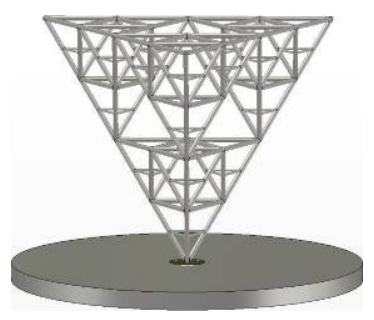

(c)

Fig. 8 Sierpinski tetrahedron antenna using, a. Solid BBS, b. Cylindrical BBS, c. Modified Cylindrical BBS

The material used for building the antennas is Perfect Electric Conductor (PEC) except the material 
that is used to interface the waveguide power source to the antenna (interfacing material) which has $\mu_{r}=1$ and $\varepsilon_{r}=3$. The settings for every antenna are shown in Fig. 9.

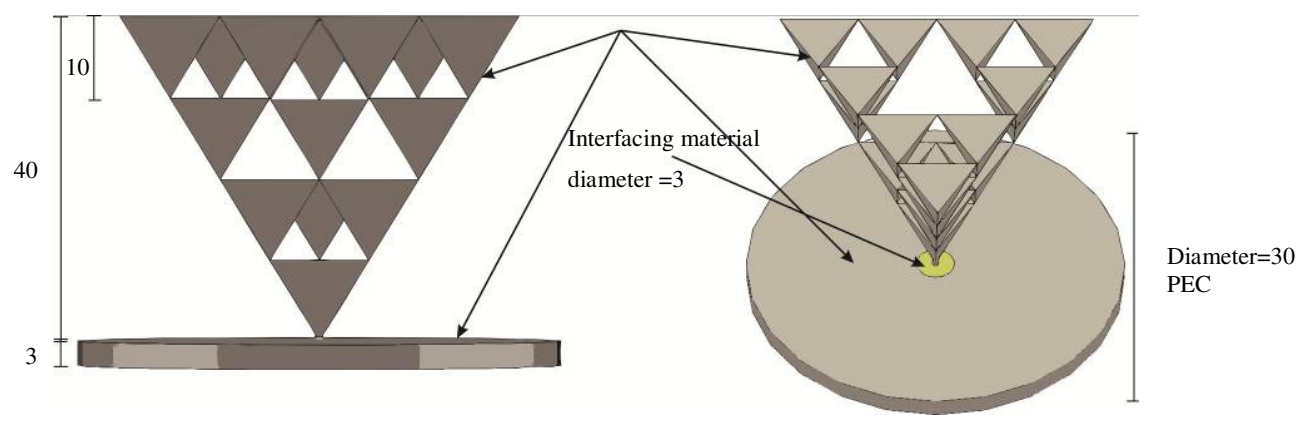

Fig. 9 Antenna dimensions and settings in $\mathrm{mm}$

A Visual Basic (VB) subroutine was built to carry out the circuit analysis inside CST studio suite. The antennas are tested for the range between $10 \mathrm{MHz}$ to $50 \mathrm{GHz}$. The Input Return Loss (IRL) curve and its phase for the antennas are shown in Fig. 10.

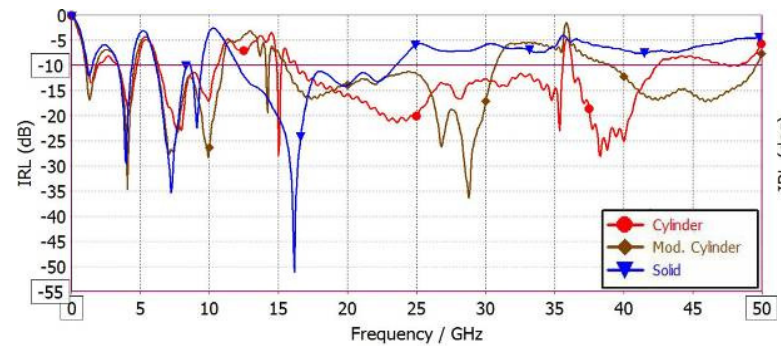

(a)

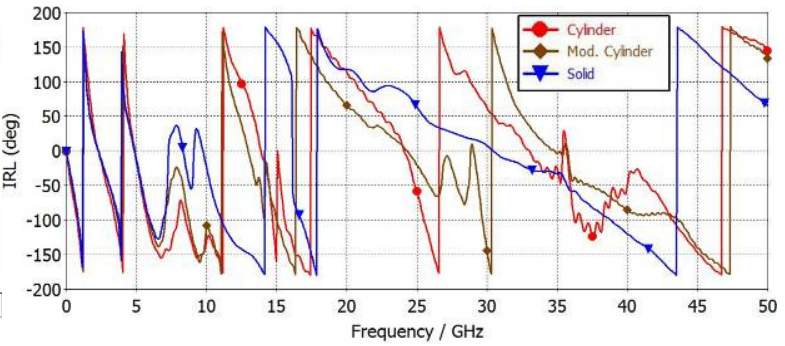

(b)

Fig. 10 The input return loss and its phase, a. Absolute IRL in dB, b. The phase

The admittance real part, imaginary part, magnitude and phase are shown in Fig.11

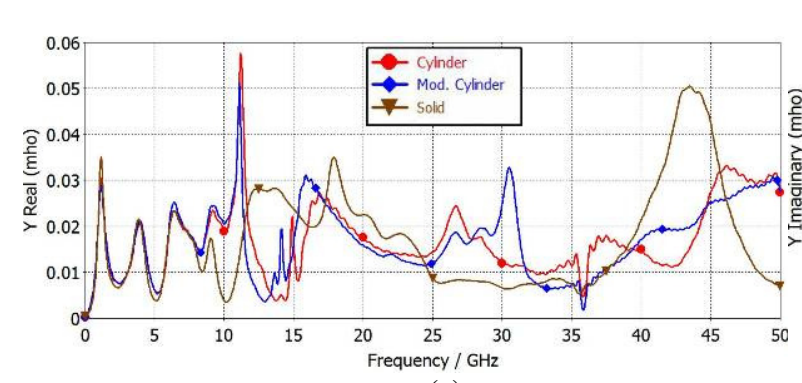

(a)

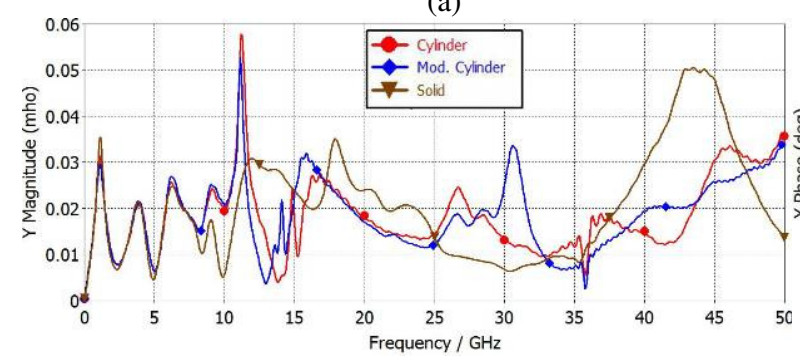

(c)

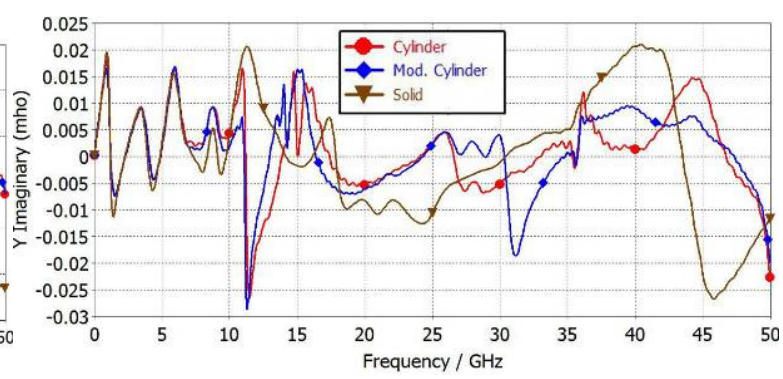

(b)

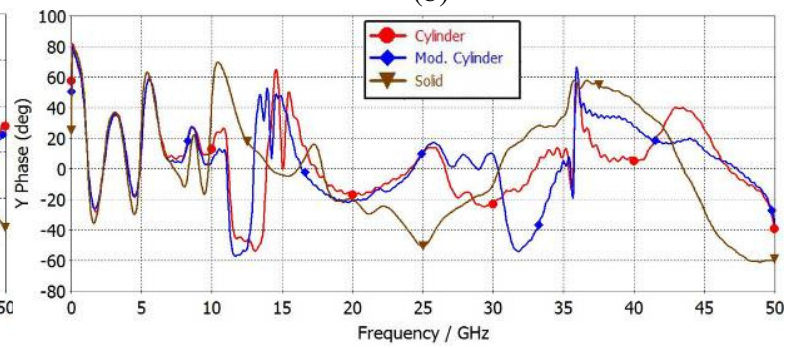

(d)

Fig. 11 The antennas impedances, a. real part, b. imaginary part, c. magnitude, d. phase

The delivered power is shown in Fig.12 


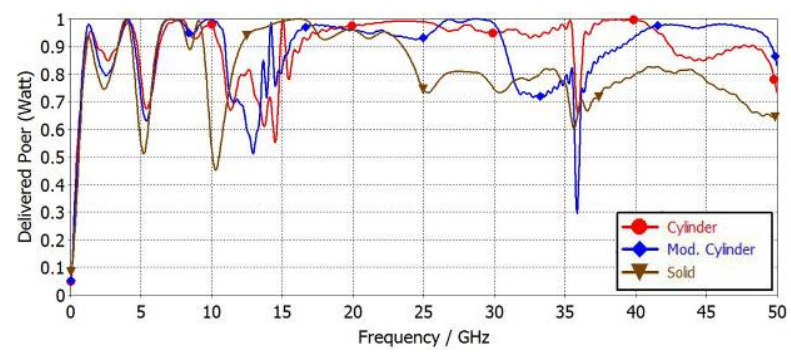

Fig. 12 The delivered power in Watt

The surface current density is an important factor to analyze the antenna parameters such as the electric field, the electric energy, the power flow, and the far field pattern. Assume that the current density is $J$ then the relation between the electric field and the current density is [36]

$$
\begin{gathered}
\vec{V}(r)=\iint_{A} \frac{\exp (-j k|R|)}{4 \pi R} J(r) d A ; \quad R=\left|r-r^{\prime}\right| \\
\vec{E}_{s}=-j \omega \mu \vec{V}(r)+\frac{1}{j \omega} \nabla(\nabla \cdot \vec{V}(r))
\end{gathered}
$$

The value of $\vec{V}(r)$ is calculated from section II. The current density at the first tuning frequency is given in Fig. 13 and for the third tuning frequency is given in Fig. 14 for the three antennas.

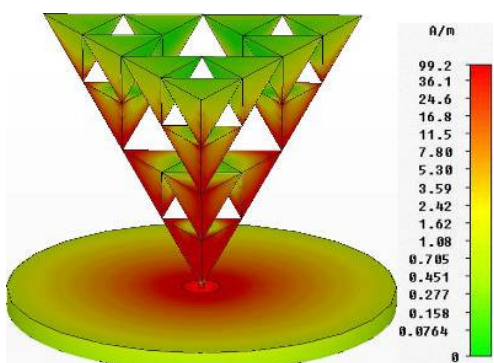

(a)

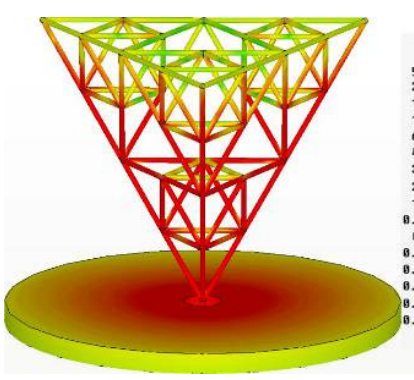

(b)
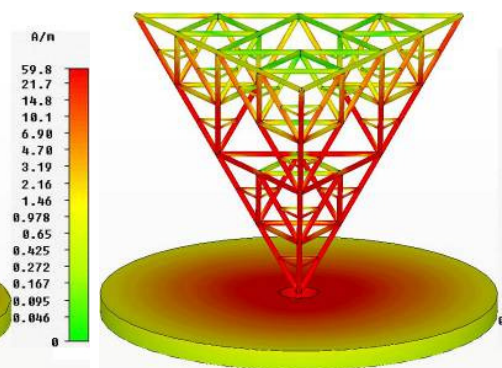

(c)

Fig. 13 The current density magnitude at the first tuning, a. Solid BBS, b. Cylindrical BBS, c. Modified Cylindrical BBS

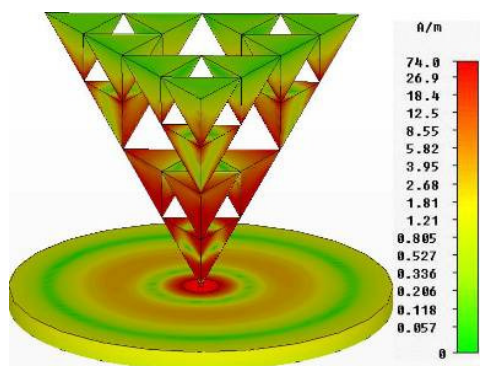

(a)

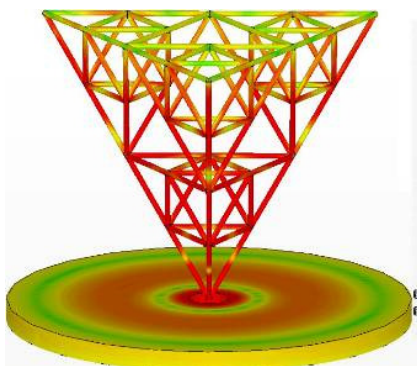

(b)
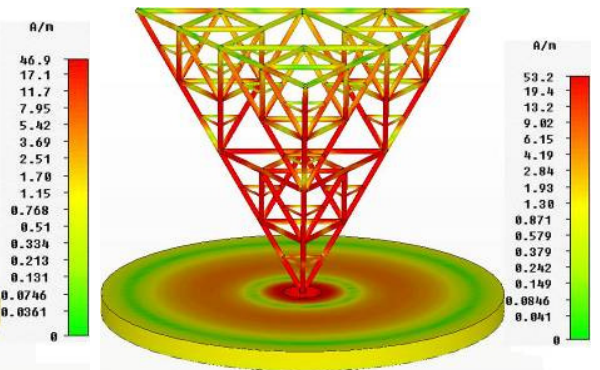

(c)

Fig. 14 The magnitude of the current density at the third tuning frequency, a. Solid, b. Cylindrical, c. Modified Cylindrical

Fig. 14 shows that the solid BBS has always higher surface current density than the other two blocks. This confirms the electrical model discussed in section II where solid BBS has higher value of $Y$. The current peak level is almost twice the current peak level of the cylindrical BBS.

The current level of the modified cylindrical BBS cannot be compared easily with the other ones because its admittance is a tandem like structure. Hence, the current peak cannot be determined easily only by using the simulation. But overall, it has lower value of $Y$ than the solid BBS and higher that the cylindrical BBS. The same can be said for the delivered power curve given in Fig. 12 and the $Y$ 
magnitude at the tuning frequencies shown in Fig.11.c in which we can see that the magnitude of the solid BBS is always below the other two BBSs.

The antennas tuning frequencies, the tuning ranges and IRL at the tuning frequency, are given in Table I.

The antennas parameters which are measured at the first three tuning frequencies are summarized in Table II.

TABLE I. ANTENNAS TUNING FREQUENCIES AND RANGES

\begin{tabular}{|c|c|c|c|c|c|c|c|c|}
\hline \multicolumn{3}{|c|}{ Solid } & \multicolumn{3}{|c|}{ Cylindrical } & \multicolumn{3}{|c|}{ Modified cylindrical } \\
\hline $\begin{array}{l}\text { Frequenc } \\
\text { y GHz }\end{array}$ & $\begin{array}{l}\text { Range } \\
\text { GHz }\end{array}$ & $\begin{array}{c}\text { IRL Level } \\
\text { dB }\end{array}$ & $\begin{array}{l}\text { Frequenc } \\
\text { y GHz }\end{array}$ & $\begin{array}{l}\text { Range } \\
\mathbf{G H z}\end{array}$ & $\begin{array}{c}\text { IRL Level } \\
\text { dB }\end{array}$ & $\begin{array}{l}\text { Frequenc } \\
\text { y GHz }\end{array}$ & $\begin{array}{c}\text { Range } \\
\text { GHz }\end{array}$ & $\begin{array}{c}\text { IRL Level } \\
\text { dB }\end{array}$ \\
\hline 1.3097 & 0.409 & -12.076 & 1.3097 & 0.463 & -17.102 & 1.3097 & 0.745 & -16.936 \\
\hline 3.9592 & 1.01 & -29.536 & 4.0592 & 0.916 & -35.431 & 4.0592 & 1.182 & -34.732 \\
\hline 7.2086 & 2.215 & -35.37 & 7.0086 & 4.248 & -24.271 & 7.0586 & 4.796 & -27.762 \\
\hline 9.108 & 0.833 & -22.521 & 26.455 & 13.192 & -21.018 & 28.804 & 15.214 & -36.401 \\
\hline 16.157 & 11.466 & -51.206 & 37.802 & 4.567 & -20.614 & 45.951 & 10.59 & -17.206 \\
\hline & & & 47.9 & 2.828 & -13.589 & & & \\
\hline
\end{tabular}

TABLE II. IMPEDANCE, IRL, VSWR, PATTERN GAIN PARAMETERS AND DELIVERED POWER FOR THE FIRST THREE TUNING FREQUENCIES

\begin{tabular}{|c|c|c|c|c|c|c|c|c|c|}
\hline & \multicolumn{3}{|c|}{ Solid } & \multicolumn{3}{|c|}{ Cylindrical } & \multicolumn{3}{|c|}{ Modified cylindrical } \\
\hline & 1.3097 & 3.9592 & 7.2086 & 1.3097 & 4.0592 & 7.0086 & $\mathbf{1 . 3 0 9 7}$ & 4.0592 & 7.0586 \\
\hline Y-Real (mho) & 0.027 & 0.021 & 0.02 & 0.025 & 0.021 & 0.012 & 0.025 & 0.021 & 0.02 \\
\hline $\begin{array}{c}\text { Y-Imaginary } \\
\text { (mho) }\end{array}$ & -0.01 & -0.001 & 0.0005 & -0.004 & -0.0003 & 0.005 & -0.004 & -0.0004 & 0.002 \\
\hline $\begin{array}{l}\text { Y-Magnitude } \\
\text { (mho) }\end{array}$ & 0.029 & 0.021 & 0.02 & 0.025 & 0.021 & 0.02 & 0.025 & 0.021 & 0.02 \\
\hline $\begin{array}{l}\text { Y-Phase } \\
\text { (Degree) }\end{array}$ & -15.915 & -2.278 & 1.508 & -10.005 & -0743 & 6.995 & -9.16 & -1.001 & 4.647 \\
\hline $\operatorname{IRL}(\mathrm{dB})$ & -12.076 & -29.536 & 2.215 & -17.102 & -35.431 & -24.271 & -16.936 & -34.732 & -27.762 \\
\hline $\begin{array}{l}\text { IRL phase } \\
\text { (Degree) }\end{array}$ & 138.09 & 143.43 & -50.59 & 141.71 & 157.5 & -92.037 & 146.27 & 151.44 & -97.399 \\
\hline VSWR & 1.663 & 1.069 & 1.035 & 1.325 & 1.034 & 1.13 & 1.332 & 1.037 & 1.085 \\
\hline $\begin{array}{l}\text { MB } \dagger \text { width } \\
\text { (Degree) }\end{array}$ & 92.3 & 55.2 & 45.5 & 92.8 & 53.1 & 40.7 & 92.6 & 53.1 & 40.1 \\
\hline $\begin{array}{c}\mathrm{MB} \dagger \text { Gain } \\
\mathrm{dB}\end{array}$ & -1.0 & 3.7 & 3.2 & -1.7 & 4.0 & 3.4 & -1.8 & 4.0 & 3.5 \\
\hline $\begin{array}{l}\mathrm{MB} \dagger \text { direction } \\
\text { (Degree) } \\
\text { Power }\end{array}$ & 89.0 & 48.0 & 80.0 & 88.0 & 45.0 & 79.0 & 88.0 & 46.0 & 79.0 \\
\hline $\begin{array}{l}\text { Delivered } \\
\text { (Watt) }\end{array}$ & 0.938 & 0.999 & 0.9997 & 0.943 & 0.985 & 0.985 & 0.98 & 0.9997 & 0.998 \\
\hline
\end{tabular}

$\dagger$ MB: Main Beam

\section{CONCLUSION}

This research introduces an iterative approach to analyze the effect of the building block geometry on the 3D pre-fractal antenna depending on the $\mathbf{Y}$ matrix. The nodal analysis represents a simple and an intuitive method to anticipate the behavior of the Sierpinski tetrahedron fractal antenna despite its complex shape. From the simulation results it can be seen that the BBS has a distinguishable effect on the antenna response. The BBS can add or subtract or alternate the value of the $\mathbf{Y}$ matrix which leads to a new distribution for the surface current density. The effect of BBS can be seen clearly at the high frequencies while at the low frequencies the BBS has no effect. At low frequencies (e.g. the first tuning frequency) when the wavelength $\lambda$ is longer than the edge length $l$ the effect of BBS geometry is not clearly obvious and all BBS's almost have the same behavior. At high frequencies (e.g. 45 
$\mathrm{GHz}$ ), the effect of BBS is obvious causing modifications to the tuning frequencies and their ranges. The effect of the BBS modification starts to take place at $9 \mathrm{GHz}$ and above when $\lambda \leq 3 \mathrm{~cm}$. This means that we can modify the antenna response at a certain frequency by adding elements that have lengths shorter than $\lambda$ so as the effect to be noticeable.

It seems that decreasing the value of $\mathbf{Y}$ yields higher number of tuning frequencies with narrower ranges as in the case of cylindrical BBS compared with the modified and solid.

The added elements to the modified cylindrical BBS result better IRL properties at the tuning frequencies compared with solid and cylindrical because we have more tuned impedances.

\section{REFERENCES}

[1] J. Lévy-Véhel and E. Lutton, Fractals in Engineering New Trends in Theory and Applications, Springer-Verlag Ltd, London 2005.

[2] K. Falconer, Fractal Geometry Mathematical Foundations and Applications, John Wiley \& Sons Ltd, England, 2003.

[3] M. Jahanbakht and A. A. Neyestanak, "Coplanar MEMS Phased Array Antenna Using Koch Fractal Geometry", Progress In Electromagnetics Research M, Vol. 17, 29-42, 2011.

[4] J.-C. Liu et. al., "Dual-mode Wide-band and Dual-band Resonators with Minkowski-Island-Based Fractal Patch for WLAN Systems", Cross Strait Quad-Regional Radio Science and Wireless Technology Conference (CSQRWC), Vol.1, 2011.

[5] P. Rao and N. Sarma, " Koch Fractal Boundary Single feed Circularly Polarized Microstrip Antenna”, Journal of Microwaves, Optoelectronics and Electromagnetic Applications, Vol. 6, No. 2, December 2007.

[6] H. Kimouche and H. Zemmour, "A Compact Fractal Dipole Antenna for 915MHz and 2.4GHz RFID Tag Applications", Progress In Electromagnetics Research Letters, Vol. 26, 105-114, 2011.

[7] G. D. Martino, et. al., "Fractal Filtering Applied to SAR Images of Urban Areas", Joint Urban Remote Sensing Event (JURSE), Munich, Germany, April 11-13, 2011.

[8] R. Kumar and P. Malathi, "Design of CPW -Fed Ultra wideband Fractal Antenna and Backscattering Reduction", Journal of Microwaves, Optoelectronics and Electromagnetic Applications, Vol. 9, No. 1, June 2010.

[9] A. Campos and Elder Eldervitch C. de Oliveira, "Design of Miniaturized Frequency Selective Surfaces Using Minkowski Island Fractal”, Journal of Microwaves, Optoelectronics and Electromagnetic Applications, Vol. 9, No. 1, June 2010.

[10] H.-X. Xu1, G.-M. Wang1, and Q. Peng2, "Fractal-Shaped Complementary Electric-LC Resonator for Bandstop Filter", Progress In Electromagnetics Research C, Vol. 23, 205-217, 2011.

[11] F. N. Najm, Circuit Simulation, IEEE John Wiley \& Sons Inc. Publication, 2010.

[12] O. Wing, Classical Circuit Theory, springer, U.S.A., 2008.

[13] Boualleg, N. Merabtine and M. Benslama, "Analysis of Fractal Radiation Patterns From Concentric-Ring Hexagonal Arrays", Semiconductor Physics, Quantum Electronics \& Optoelectronics, Vol. 8, No. 4, pp. 91-94, 2005.

[14] C. Borja, C. Puente, and A. Medina, "Iterative Network Model to Predict the Behavior of a Sierpinski Fractal Network", IEEE Electronic Letters, Vol. 4, No. 15, 23rd July, 1998.

[15] C. P. Baliarda et. al., "An Iterative Model for Fractal Antennas: Application to the Sierpinski Gasket Antenna", IEEE Transactions on Antennas and Propagation, Vol. 48, No.5, 2000.

[16] Adrover et. al, "Circuit Models of Fractal-Geometry Antennas", Atti della XIV RiNEm, Ancona, 16-19 September , pp. 468-471, 2002.

[17] Nasimuddin and A. K. Verma, "Fast and Accurate Model for Analysis of Equilateral Triangular Patch Antenna", Journal of Microwave and Optoelectronics, Vol. 3, No. 4, 2004.

[18] J. S. Belrose, "A Wire Model for the Sierpinski Gasket Type Monopole Antenna and a truly Broadband Bent-Wire Antenna", IEEE Antennas and Propagation Society International Symposium, 2004.

[19] W. Arrighetti, P. De Cupis, and G. Gerosa, "Circuit Models for Sierpinski gasket antennas" Available at arXiv:physics/0510069v1, 2005.

[20] F. Abdelhak et. al., "Analysis and Design of Printed Fractal Antennas by Using an Adequate Electrical Model", International Journal of Communication Networks and Information Security (IJCNIS), Vol.1, No. 3, 2009.

[21] M. Alaydrus, "Analysis of Three Dimensional Sierpinski Gasket Antennas", Indonesia-Malaysia Microwave Antenna Conference. Depok, 101-104, 2010.

[22] M. Alaydrus, "Analysis of Sierpinski Gasket Tetrahedron Antennas", Journal of Telecommunications, Vol. 5, Issue 2, 2010.

[23] S.-T. Fan et. al., "A Novel Self-Similar Antenna for UWB Applications with Band-Notched Characteristics", Progress In Electromagnetics Research Letters, Vol. 22, 1-8, 2011.

[24] L. Lazareck, G. Verch, and J. F. Peters, "Fractals in Circuits", Canadian Conference on Electrical and Computer Engineering, Vol. 1, Page(s) 589 - 594, 2001.

[25] U. S. Inan and R. A. Marshall, Numerical Electromagnetics The FDTD Method, Cambridge University Press, N.Y., 2011. 
[26] S.-Q. Xiao, Z. H. Shao, and B.-Z. Wang, "Application of the Improved Matrix Type FDTD Method for Active Antenna Analysis", Progress In Electromagnetics Research, PIER 100, 245-263, 2010.

[27] M. Weiner, Electromagnetic Analysis Using Transmisson Line Variables, World Scientific Publishing Co. Pte. Ltd, Singapore, 2001.

[28] C. Christopoulos, The Transmission-Line Modeling (TLM) Method in Electromagnetics, A Publication in the Morgan \& Claypool Publishers series, 2006.

[29] P. Dan-Klang and Leelarasmee E., "Simulation of Voltage and Current Distributions in Transmission Lines Using State Variables and Exponential Approximation", ETRI Journal, Vol. 31, No. 1, February 2009.

[30] R. Burioni, D. Cassi and F. M. Neri, "AC Properties of 3d Sierpinski Gaskets: Rigorous Results", Journal of Physics A: Mathematical and General, Vol. 38, No. 14, April 2005,

[31] M. Niknejad, "Two-Port Networks and Amplifiers", Berkeley Wireless Research Center, University of California, Berkeley, September 22, 2008.

[32] D. Fricky, "Conversion Between S, Z, Y, h, ABCD, and, T Parameters which are Valid for Complex Source and Load Impedances", IEEE Transaction on Microwave Theory and Techniques, Vol. 42, No. 2, Feb. 1994.

[33] A.-S. BIN JA'AFAR, "Sierpinski Gasket Patch and Monopole Fractal Antenna", M. Sc. Thesis in Electrical-Electronics \& Telecommunications, Faculty of Electrical Engineering Universiti Teknologi Malaysia, 2005

[34] "S-Parameter Design", Agilent Technologies application note 154, 2000.

[35] J. Van Bladel, Electromagnetic Fields, JohnWiley \& Sons, Inc. Hoboken, 2nd Ed., New Jersey, 2007.

[36] S. Orfanidis, Electromagnetic Waves and Antennas, Rutgers University, 2002. 